\title{
Recycling von Lithium-lonen-Batterien: Herausforderungen und aktuelle Forschungsergebnisse
}

\author{
Stefan Windisch-Kern ${ }^{1}$, Alexandra Holzer ${ }^{1}$, Christoph Ponak ${ }^{1}$, Peter Nagovnak ${ }^{2}$ und Harald Raupenstrauch ${ }^{1}$ \\ 'Lehrstuhl für Thermoprozesstechnik, Montanuniversität Leoben, Leoben, Österreich \\ ${ }^{2}$ Lehrstuhl für Energieverbundtechnik, Montanuniversität Leoben, Leoben, Österreich
}

Eingegangen 19. Januar 2021; angenommen 3. Februar 2021; online publiziert 1. März 2021

\begin{abstract}
Zusammenfassung: Der Einsatz von wertvollen und teilweise kritischen Rohstoffen wie Kobalt, Nickel, Mangan und Lithium in Kathodenmaterialien sowie die prognostizierten Marktentwicklungen machen das Recycling von Lithium-Ionen-Batterien zu einem abfallwirtschaftlich relevanten Thema. Dieser Beitrag beleuchtet die Entwicklung und Vielfalt dieser Kathodenmaterialien und leitet daraus Anforderungen an zukünftige Aufbereitungs- bzw. Recyclingverfahren ab. Die schnelle Weiterentwicklung der Zellchemismen hin zu nickelreichen Kathodenmaterialien stellt bestehende Verfahren vor wirtschaftliche Probleme und unterstreicht zusätzlich die Notwendigkeit eines flexiblen Prozesses, welcher mit einer variierenden chemischen Zusammensetzung des Abfallstromes zurechtkommen muss. Zusätzlich wird ein umfassender Einblick in die diesbezüglichen Forschungsbestrebungen des Lehrstuhls für Thermoprozesstechnik gegeben. Die präsentierten Ergebnisse des eingesetzten Verfahrenskonzepts attestieren dem Ansatz ein großes Potenzial. Beispielsweise gelang es, die als Bottleneck der Pyrometallurgie eingestufte Verschlackung von Lithium, großteils zu unterbinden und über $90 \%$ des Elements aus dem experimentellen Reaktor zu entfernen.
\end{abstract}

Schlüsselwörter: Lithium-lonen-Batterien, Recycling, Verfahrensentwicklung, Metallrückgewinnung

Recycling of Lithium-Ion-Batteries: Challenges and Latest Research Results

Abstract: Due to their properties, lithium-ion batteries (LIB) are used in a wide range of applications, including mobile electronics, electromobility, and stationary storage systems. Each of these applications has different requirements for the battery used, which leads to a variety of LIB types

Dipl.-Ing. S. Windisch-Kern ( $\bowtie)$

Lehrstuhl für Thermoprozesstechnik,

Montanuniversität Leoben,

Franz Josef-Straße 18,

8700 Leoben, Österreich

stefan.windisch-kern@unileoben.ac.at that differ in their cell chemistry, structure, and properties. The choice of the cathode material, which mostly consists of a lithium metal oxide structure and is subject to continuous development, has a particularly large influence on the properties of a cell. Since metals, such as cobalt, nickel, manganese, or lithium used in these cathode materials, are valuable and partly critical raw materials, the recycling of LIBs is of great relevance. Yet, because of the fast-evolving cell chemistries, the future waste stream is expected to be very dynamic and poses a challenge for recycling processes. This article describes the development and diversity of cathode materials and derives requirements for future treatment and recycling processes. The rapid development of LIB cells towards nickel-rich cathode materials causes economic problems for existing processes and points out the need for a flexible treatment which has to cope with a varying chemical composition of the future waste stream. In addition, a comprehensive insight into the relevant research efforts of the Chair of Thermal Processing Technology is given. The presented results of the applied reactor concept attest the approach a great potential. For example, the slagging of lithium, which is considered as a bottleneck in pyrometallurgical LIB recycling, was largely prevented and over $90 \%$ of the lithium were removed from the experimental reactor.

Keywords: Lithium-ion batteries, Recycling, Process development, Metal recovery

\section{Einleitung}

In der Individualmobilität von morgen wird dem Elektroauto eine bedeutende Rolle vorhergesagt. Sowohl die Dekarbonisierungsziele als auch der nationale und EU-verbindliche Klima- und Energieplan, welcher im Dezember 2019 vorgestellt wurde, sehen eine CO2-Einsparung im Verkehrssektor von mindestens $30 \%$ bis 2030 vor. Der Anteil erneuerbarer Energien im Verkehrssektor soll bis dahin auf $14 \%$ gewachsen sein [1]. Da Lithium-Ionen-Batterien (LIBs) 
über einige überzeugende Vorteile gegenüber anderen Batterie-Technologien verfügen, wie zum Beispiel eine höhere Energie- und Leistungsdichte sowie größere Ladungshübe, wird erwartet, dass diese in einem bedeutenden Anteil der prognostizierten Elektromobilität Verwendung finden. Diese Elektromobilität wird in den mittelfristigen Strategiepapieren der Europäischen Kommission als wichtige Säule propagiert, wobei je nach angewandtem Szenario beträchtliche Unterschiede in der Entwicklung des LIB-Bedarfs beobachtet werden können. In sogenannten "LOW“-Szenarien wird davon ausgegangen, dass sich weder politisch noch gesellschaftlich im Vergleich zum Basisjahr 2018 eine Veränderung einstellt. Dies erscheint im Lichte der letzten politischen Entwicklungen und dem Aufschwung der GrünParteien in einigen europäischen Staaten bei den letzten Wahlen jedoch unwahrscheinlich. Die als "moderate" betitelten Vorhersagemodelle, welche davon ausgehen, dass die Entwicklung von Elektromobilität mit LIB-Technologie rein durch die ökologische Notwendigkeit im Kampf gegen den Klimawandel weitergetrieben wird, gehen bereits von mehreren hundert Millionen Elektroautos (BEV und PHEV zusammen) bis zum Jahr 2050 aus. Im Kielwasser der Technologiefortschritte für die Elektromobilität könnten auch stationäre Energiespeichersysteme im Hinblick auf eine optimierte Einbindung erneuerbarer Energieträger eine signifikante Ausweitung erfahren. Eine solche Entwicklung, deren Vorhersage-Graphen in ihren Trends jenen der Elektromobilität ähneln, brächte eine weitere Erhöhung des LIB-Einsatzes mit sich. Während die Absatz-Richtung angesichts globaler politischer Anstrengungen im Kampf gegen den Klimawandel also einigermaßen klar erscheint und den LIBs eine erfolgreiche Zukunft vorhergesagt werden kann, ist die Gewissheit um die tatsächliche Zellzusammensetzung der Lithium-Ionen-Batterien in zukünftigen Elektroautos weitaus geringer. Daher ist es notwendig, über die Behandlung und Wiederverwendung in die Jahre gekommener zukünftiger Automobile und deren Batterien nach-

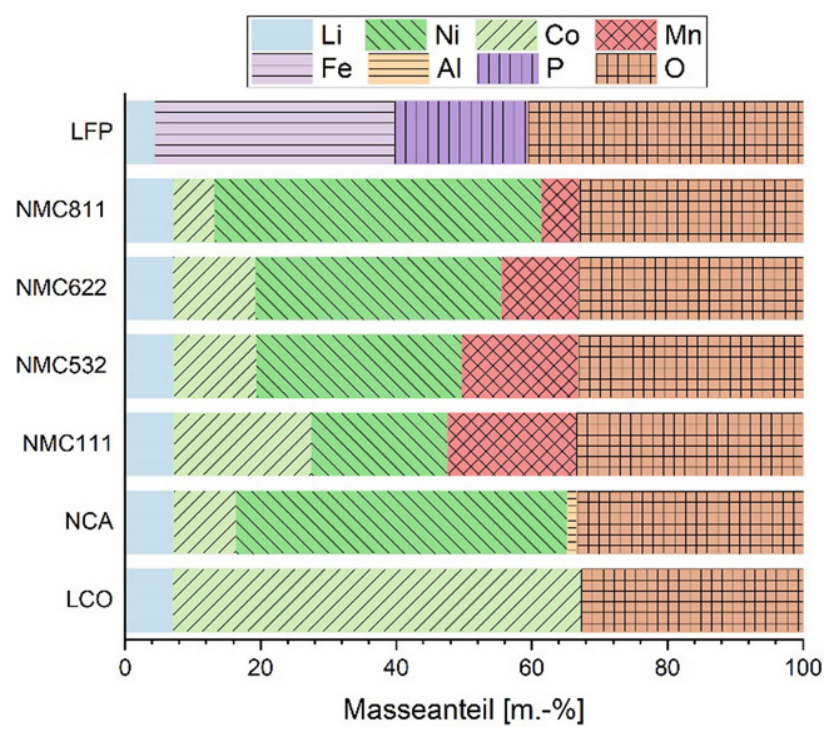

Abb. 1: Rohstoffanteil in Massenprozent (m.-\%) nach Kathodentechnologie zudenken und geeignete wirtschaftliche Verwertungs- und Wiederverwendungsmöglichkeiten zu finden.

\section{Lithium-Ionen-Batterien: Eine Bezeich- nung - aber viele Technologien}

Aufgrund der grundsätzlichen Arbeitsweise von LithiumIonen-Batterien kommt eine Vielzahl an Kombinationsmöglichkeiten von Elektroden- und Elektrolytmaterialien in Frage. Die grundsätzlichen Technologieunterschiede sind dabei vor allem im eingesetzten Kathodenmaterial zu finden. Im Bereich der Elektromobilität wird das Feld von derzeit drei Zellchemismen angeführt, welche nachfolgend kurz beschrieben werden.

a) NCA - Lithium-Nickel-Kobalt-Aluminium-Oxid $\left(\mathrm{Li}\left(\mathrm{Ni}_{x} \mathrm{Co}_{y} \mathrm{Al}_{1-\mathrm{x}-\mathrm{y}}\right) \mathrm{O}_{2}\right)$

NCA ist seit den späten 1990er-Jahren bekannt und bietet Vorteile bei spezifischer Energie sowie Leistung. Nachteilig ist dabei jedoch die Sicherheit der Zelle, welche besonderer Überwachungsmechanismen beim Einsatz in Elektroautomobilen bedarf. Trotz ihres vergleichsweise geringen Kobalt-Einsatzes ist die Herstellung von NCA-Zellen teuer, was ihre Verbreitung in der Automobilindustrie stark behindert [2].

b) NMC - Lithium-Nickel-Mangan-Kobalt-Oxid (Li(NixMnyCo1-x-y)O2)

Der Vorteil von NMC liegt in der Möglichkeit, durch gezielten Einsatz des Verhältnisses aus Nickel, Mangan und Kobalt verschiedene Zelleigenschaften produzieren zu können. Während die Kosten durch den reduzierten Einsatz von Kobalt verringert werden können, erhöht der Einsatz von Nickel die spezifische Energiedichte. Mithilfe eines größeren Anteils an Mangan lässt sich wiederum die spezifische Leistung erhöhen [2]. Diese Variabilität ist es, welche NMC eine größere Attraktivität für weitere Forschungsarbeit beschert und auf lange Sicht in die Lage versetzt, NCA nachhaltig vom Markt zu verdrängen.

c) LFP - Lithium-Eisen-Phosphat (LiFePO4)

Lithium-Eisen-Phosphat-Zellen punkten mit ihrer hohen intrinsischen Sicherheit sowie der erhöhten Leistungsdichte. Die bessere Verfügbarkeit der im Zellverbund verwendeten Elemente verbilligt die Herstellung. Nachteilig wirkt sich bei Zellen dieses Typs jedoch ihre schnellere Selbstentladung, welche nur zum Teil durch kostspielige Balancing-Systeme ausgeglichen werden kann, sowie die im Vergleich zu NMC und NCA deutlich geringere Energiedichte aus.

\subsection{Kosten als Schlüsselfaktor im Wettstreit der Technologien}

Abb. 1 zeigt einen Vergleich der Zusammensetzungen einiger LIB-Technologiemöglichkeiten im Bereich der Elektromobilität. Das zuunterst angeführte LCO (Lithium-Kobalt-Oxid) findet aufgrund seiner hohen Energiedichte, langen Lebensdauer und leichter Produzierbarkeit vor allem in 


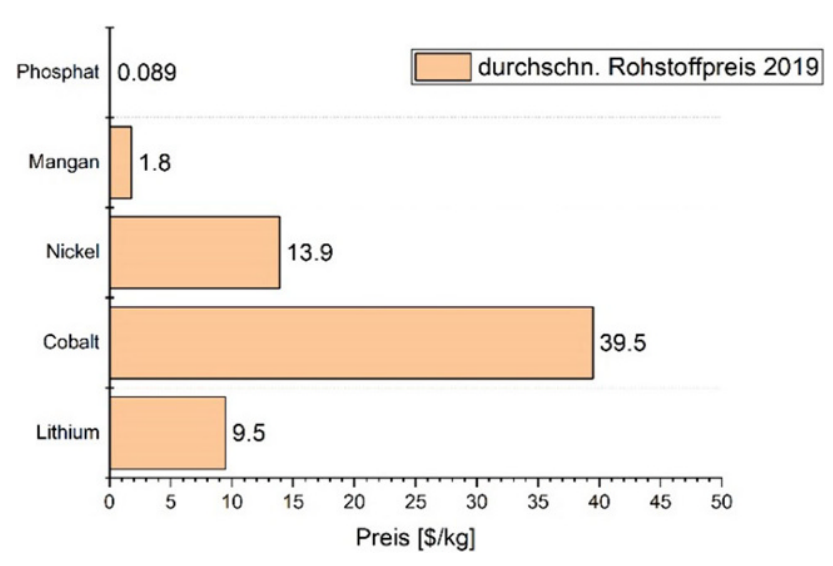

Abb. 2: Durchschnittliche Rohstoffpreise 2019 pro Kilogramm. (Nach [4])

Produkten der modernen Unterhaltungselektronik Verwendung. Auffällig ist, dass das der Technologie den Namen verleihende Lithium in vergleichsweise geringen Massen von rund $7 \mathrm{~m} . \%$ in NCA, LCO und den NMC Varianten bzw. sogar unter $5 \mathrm{~m} .-\%$ in LFP vorkommt.

Da aktuelle Batterietechnologien, und in weiterer Folge Elektroautos insgesamt, im Vergleich mit herkömmlichen, mit Verbrennungsmotoren ausgestatteten Automobilen noch immer teuer sind, wird laufend nach kostengünstigeren Ersatztechnologien geforscht. Um die Kosten auf die von der EU-Kommission erhofften $75 € / \mathrm{kWh}$ bis 2030 reduzieren zu können, muss der Anteil an Kobalt so gering wie möglich gehalten werden [3]. Abb. 2 gibt eine Übersicht über das Ungleichgewicht der eingesetzten Materialien bei den Kosten je Kilogramm. Diese sind neben der Verbesserung der elektrochemischen und physikalischen Eigenschaften der Batterien der Hauptgrund für die derzeit herrschenden Verbesserungsanstrengungen am Technologiemarkt. Der Anteil von Kobalt an den gesamten Materialkosten der Zelle ist mit einem durchschnittlichen Rohstoffpreis im Jahr 2019 von etwa 40 US-\$/kg im Vergleich zu Nickel, Mangan oder Lithium überdurchschnittlich hoch und war in der Vergangenheit immer wieder starken Anstiegen und Abfällen unterworfen. Beispielsweise stand der Preis für ein Kilogramm Kobalt im April 2018 zwischenzeitlich bereits bei rund 110 US-\$ [4]. Die neben Lithium im Einsatz stehenden Bestandteile von LFP-Zellen, Phosphat und Eisen, sind im Vergleich zu den in NCA und NMC eingesetzten Materialien um ein Vielfaches günstiger. Bis vor Kurzem waren Batterien mit Kathodenmaterial aus Lithium-Eisenphosphat die bei weitem verbreitetste Lithium-lonen-Technologie in chinesischen Elektrofahrzeugen. Weltweit befahren $88 \%$ der elektrischen Verkehrsbusse mit diesen sogenannten LFPBatterien die Straßen ihrer Kommunen und Städte mit dem Vorteil, schnell die günstig angeschafften Batterien an dafür ausgerüsteten Haltestellen nachladen zu können und damit den Nachteil der geringeren Reichweite zu überdecken [3].

Derzeit weisen die Ergebnisse vieler Studien jedoch darauf hin, dass die NMC-Kathodentechnologie in Zukunft eine zentrale Rolle spielen wird, wobei die genaue stöchiometrische Zusammensetzung der Technologie umstritten ist. Während das Consulting-Büro Avicenne [5]Energy im Jahr 2030 noch einen ausgeglichenen Mix aus verschiede- nen Zusammensetzungen voraussieht, gehen die Analysten von Bloomberg New Energy Finance [6] davon aus, dass das sich derzeit noch im Entwicklungsstadium befindende und aufgrund seines geringeren Kobalt-Gehalts günstigere NMC-811 bis dahin aktuell marktreife Technologien bereits verdrängt haben wird. Das zurzeit in Tesla-Fahrzeugen verbaute NCA wird aufgrund verschiedener nachteiliger Faktoren auf längere Frist gesehen wahrscheinlich an Relevanz verlieren. Mit einem Tesla-Marktanteil von $17 \%$ am gesamten deutschen Elektroauto-Markt bei den Neuzulassungen 2019 bzw. knapp 10\%, wenn auch Plug-In Hybride miteinbezogen werden, wird der zu erwartende Abfallstrom jedoch gerade in den nächsten zehn Jahren einen hohen NCA-Anteil im zu rezyklierenden Abfallstrom aufweisen [7].

\section{Anforderungen an Recyclingverfahren}

Die Menge an in Zukunft zu erwartenden Energiespeichern aus dem Elektromobilitätssektor und der darin zum Einsatz kommenden hochwertigen Metalle bereitet einem Recyclingverfahren, welches diese Rohstoffe für einen erneuten Einsatz in Energiespeichern wiedergewinnen kann, einen fruchtbaren Boden. Da die eingesetzten Metalle teils spärlich bis gar nicht in Europa vorkommen und deren Abbau nicht in allen Fällen ökologisch oder sozial nachhaltig ist, ist die Entwicklung eines entsprechenden Verfahrens von großer Bedeutung. Erschwerend steht dieser Entwicklungsaufgabe entgegen, dass der zukünftige Abfallstrom keinesfalls definiert ist und ein zu entwickelndes Verfahren mit sich verändernden Abfallzusammensetzungen, bestehend aus unterschiedlichen LIB-Technologien in differenzierten Verhältnissen, zurechtkommen muss, um wirtschaftlich bestehen zu können. Die Vielzahl an verbauten Materialien in einem komplexen, fest verbauten und geschützten Gehäuse birgt zusätzliche Herausforderungen, welche sowohl die Machbarkeit als auch Rentabilität der Recyclingtechniken beeinflussen können. Auch wenn Lithium, wie oben erwähnt, mit nur geringen Massen in den LIBs verbaut ist, kann davon ausgegangen werden, dass durch die in Zukunft zu erwartenden hohen Mengen an EVs bzw. End-of-Life LIBs (EoL-LIBs), auch die Gewinnung von primärgradigem Lithium zu einem wichtigen Bestandteil des Batterie-Recyclings werden wird. Mehrere Studien, welche in [8] zusammengefasst wurden, stufen die Verfügbarkeit über die nächsten Jahrzehnte aufgrund der vorhergesagten Nachfragesteigerung am Elektroautomobilmarkt als kritisch ein. Das Fraunhofer-Institut für Systemund Innovationsforschung (ISI) [9] sieht beispielsweise bei entsprechend positiver Entwicklung des Elektroautoabsatzes ohne Verringerung des Lithiumanteils bzw. veränderter Zellchemie eine mögliche Übersteigung der derzeit bestehenden Lithiumreserven bis ins Jahr 2050. Auch bei späterer flächendeckender Verbreitung der Elektromobilität ist ein starker Anstieg des Lithiumbedarfs vorauszusehen. 


\subsection{Aktuelle und zukünftige Recyclingmöglich- keiten}

Grundsätzlich können LIB-Zellen sowohl durch hydro- als auch pyrometallurgische Prozesse rezykliert werden. Während bei pyrometallurgischen Prozessen versucht wird, die als Oxide vorliegenden Metalle thermochemisch zu reduzieren und dadurch für einen weiteren Einsatz zurückzugewinnen, verfolgen hydrometallurgische Verfahren dieses Ziel mittels Lösungsmittelextraktion, Elektrolyse und chemischen Fällungsreaktionen [10]. Der Stand der Technik bisheriger Recycling-Anstrengungen auf pyrometallurgischer Basis, wie das in der Literatur oft zitierte Umicore-Verfahren [11], begnügt sich nach der Vorbehandlung bzw. Zerlegung der Batterien mit der Herstellung einer Metall-Legierung aus Kupfer, Kobalt, Nickel und Eisen, während Lithium in der Schlacke gebunden und nicht mehr im "Closed-loop" rückgeführt wird, weil eine hydrometallurgische Extraktion mittels Lösungsmitteln aus der Schlacke, obwohl technisch möglich, bislang nicht wirtschaftlich war. Laut einem Bericht der Europäischen Kommission vom April 2019 wurde erst vor Kurzem damit begonnen, auch Lithium aus der Schlacke für eine Wiederverwendung zu gewinnen [12]. Bisherige marktreife Recyclingprozesse auf pyro- oder hydrometallurgischer Basis teilen alle den Nachteil, nur wirtschaftlich operieren zu können, solange der Anteil an teurem Kobalt und Nickel im Einsatzmaterial hoch ist. Aluminium, Lithium, und andere billigere Metalle, deren Reduktion oder Extraktion derzeit noch nicht flächendeckend wirtschaftlich ist, verbleiben im Reststoff - dessen Verwertung in der Baustoffindustrie kein wünschenswertes Endergebnis für die Zukunft sein kann. Da sich die Kobaltkonzentrationen in zukünftigen LIBs reduzieren werden, hat ein Recyclingverfahren, welches in Zukunft nicht nur Kobalt und Nickel, sondern alle werttragenden Metalle aus dem Kathodenmaterial der Lithium-lonen-Batterien zurückgewinnen kann, einen enormen und über die Einsatzdauer von Lithium-Ionen-Batterien nachhaltigen, wirtschaftlichen Vorteil gegenüber Konkurrenzverfahren.

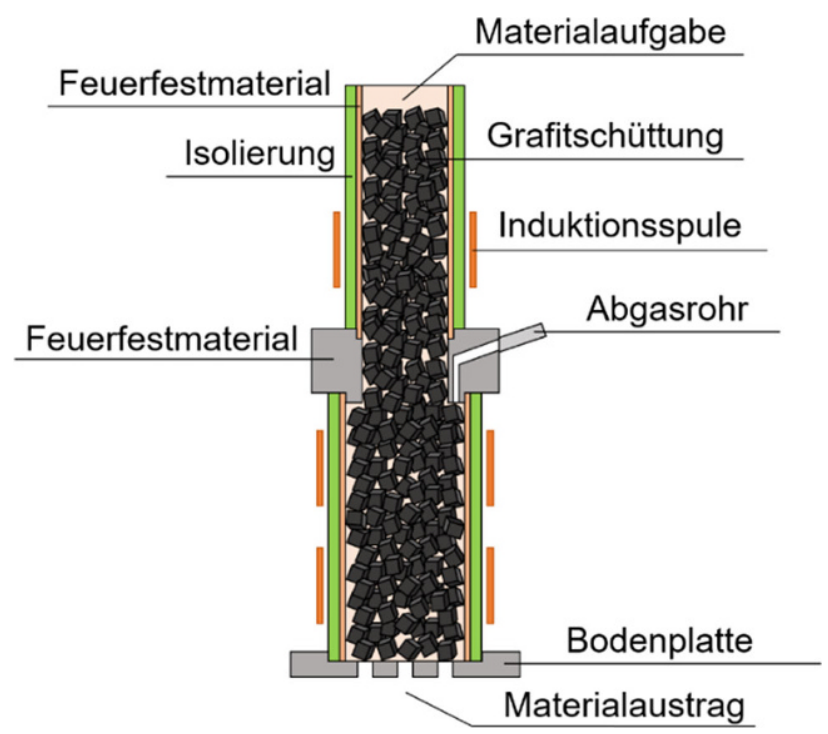

Abb. 3: Schematische Darstellung des InduRed-Reaktors [13]

\section{Forschung am Lehrstuhl für Thermo- prozesstechnik der Montanuniversität Leoben}

Am Lehrstuhl für Thermoprozesstechnik der Montanuniversität Leoben wird aus genannten Gründen an der Entwicklung eines pyrometallurgischen Recyclingverfahrens gearbeitet, welches alle Wertmetalle aus dem Kathodenmaterial zurückgewinnen kann. Dabei soll der sogenannte InduRed-Reaktor (Abb. 3) zum Einsatz kommen, welcher ursprünglich für die Rückgewinnung von Phosphor aus Klärschlammaschen konzipiert wurde $[13,14]$ und dank seiner spezifischen Eigenschaften wie dem geringen Sauerstoffpartialdruck, der Möglichkeit gasförmige Reaktionsprodukte punktuell abzuziehen und der durch die Grafitwürfelschüttung bereitgestellten großen Reaktionsoberfläche ein großes Potenzial aufweist. Ziel ist es, in dem induktiv beheizten Schüttschichtreaktor alle Wertmetalle aus zuvor aufbereiteten LIBs aus ihren oxidischen Verbindungen zu reduzieren und dabei simultan die Rückgewinnung des bislang unrentablen, aber in Zukunft verstärkt nachgefragten Lithiums zu ermöglichen. Das Verfahrenskonzept sieht vor, Schwarzmasse („Black Matter"), ein wertstoffreiches Zwischenprodukt der LIB-Recyclingkette, mit etwaigen Zuschlagstoffen von oben in den kontinuierlich arbeitenden Reaktor zu chargieren und schrittweise zu erhitzen, zu schmelzen und zu reduzieren. Durch die stark reduzierenden Bedingungen im Reaktor soll dabei die ansonsten für pyrometallurgische Ansätze typische Verschlackung von Lithium unterbunden und das Element stattdessen als Teil der Gasphase aus dem Reaktor entfernt werden. Die restlichen Wertmetalle werden ebenfalls reduziert und als Legierung, im Fall von NCA und NMC bestehend aus Nickel, Kobalt, Mangan und Aluminium, separat zurückgewonnen.

Dass eine grundsätzliche Adaptierung des Reaktorkonzepts für andere Reststoffe als Klärschlammasche möglich ist, hat bereits Ponak $[15,16]$ gezeigt, in dessen Arbeit das Portfolio des Reaktors um dessen Anwendung auf Stahlwerksschlacken erweitert wurde. Um zu untersuchen, ob sich das Konzept auch für die Behandlung von Zwischenprodukten der LIB-Recyclingkette eignet und ob bzw. in welcher Form Anpassungen am Reaktor nötig sind, untersucht ein Team des Lehrstuhls für Thermoprozesstechnik seit Mitte 2019 die Eigenschaften von LIB-Kathodenmaterialien unter reduzierenden Bedingungen. Dazu werden die Materialien LCO, NCA, LFP sowie NMC in den Varianten 111, 532, 622 und 811 mit in unterschiedlichen Analysegeräten bzw. Versuchsanlagen auf bis zu $1650^{\circ} \mathrm{C}$ erhitzt, um deren Verhalten bei hohen Temperaturen zu untersuchen. Um reduzierende Bedingungen einzustellen werden die Proben, je nach eingesetztem Gerät, mit 10-25 m.-\% Kohlenstoffpulver versetzt. Der erste Schritt zur Charakterisierung der pulverförmigen Materialien passiert dabei in einem Erhitzungsmikroskop, in welchem die in Zylinderform gepressten Proben auf $1650^{\circ} \mathrm{C}$ erhitzt und währenddessen ihre Querschnittsflächen aufgezeichnet werden. Durch Beobachtung der Änderungen der Querschnittsfläche können Temperaturbereiche ausfindig gemacht werden, in welchen Reaktionen, Phasenänderungen oder Schmelzvorgänge stattfinden, die diese Änderungen hervorrufen. Eine 

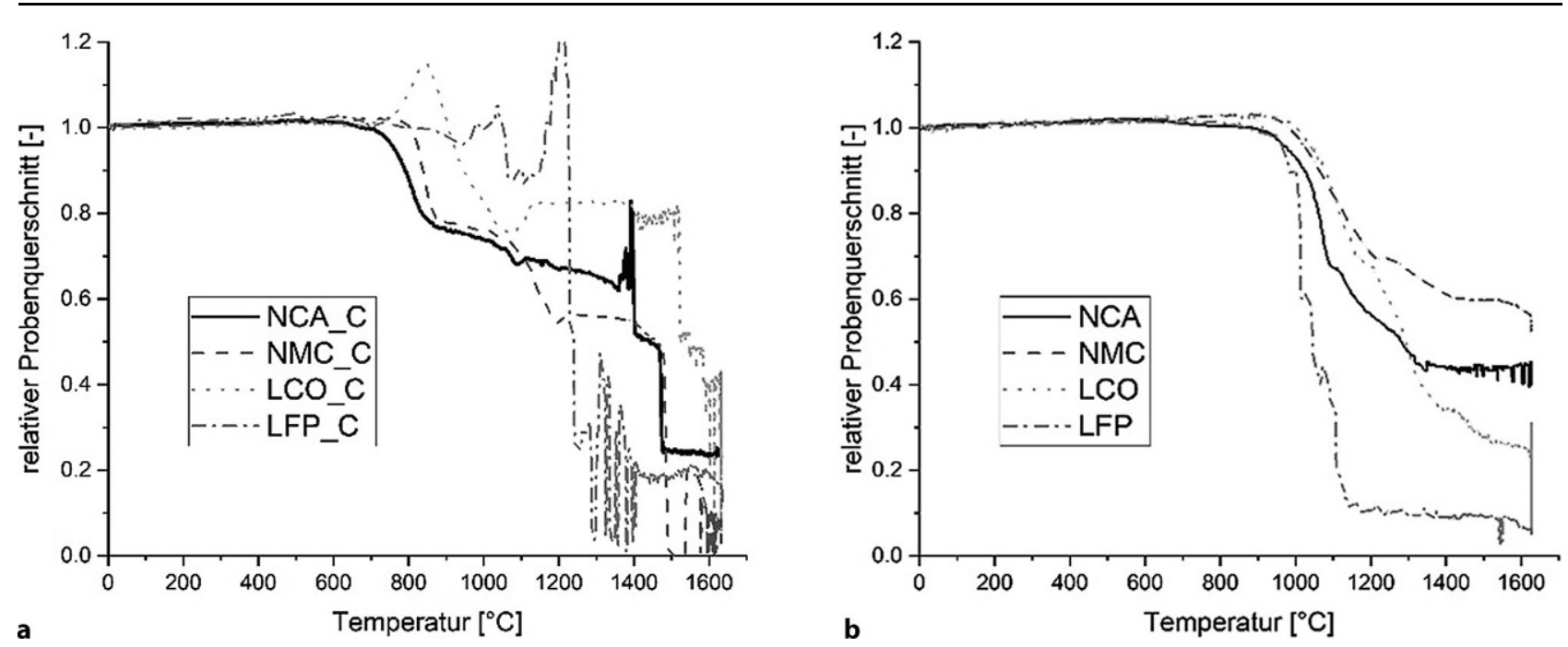

Abb. 4: Gegenüberstellung der Ergebnisse aus den Erhitzungsmikroskop-Versuchen aktueller Kathodenmaterialen (a) mit und (b) ohne Kohlenstoffzugabe [15]
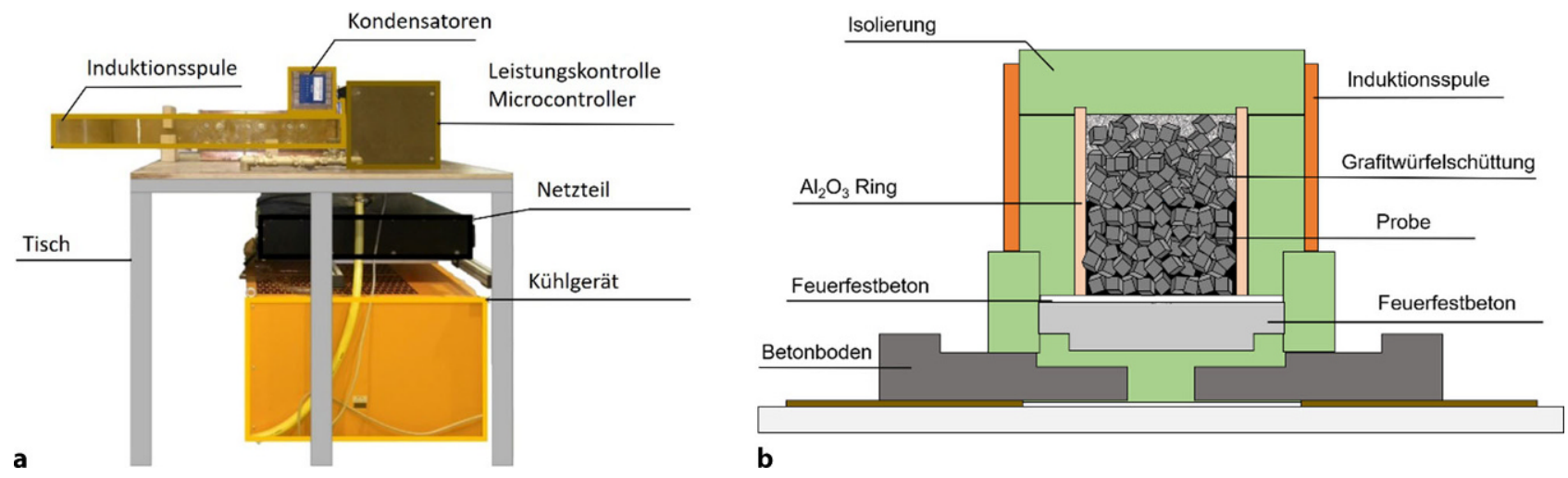

Abb. 5: a Aufbau der InduMelt-Anlage und (b) schematische Darstellung des eingesetzten Tiegelkonzepts $[13,14]$

detaillierte Beschreibung der Durchführungs- und Interpretationsmethodik dieser Analysen wurde von den Autoren auch in [17] bereits veröffentlicht. Ein Überblick über die wichtigsten Ergebnisse dieser Erhitzungsmikroskop-Versuchsreihe ist Abb. 4 zu entnehmen, in der die Trends der Querschnittsflächen von LCO, NCA, NMC und LFP jeweils mit und ohne Kohlenstoffzugabe dargestellt sind.

Beim direkten Vergleich der beiden Graphen sind deutliche Unterschiede erkennbar. Zum einen treten die ersten signifikanten Änderungen in den Proben mit Kohlenstoffzugabe (Abb. 4a) deutlich früher, nämlich bei rund $800^{\circ} \mathrm{C}$, auf, während sie bei Versuchen ohne Kohlenstoffzugabe (Abb. 4b) erst bei rund $1000^{\circ} \mathrm{C}$ zu beobachten sind. Zum anderen ist bei Versuchen mit Kohlenstoffzugabe ein weiterer auffälliger Temperaturbereich bei rund $1500^{\circ} \mathrm{C}$ zu erkennen, wobei die Querschnittsfläche noch einmal sprunghaft abnimmt und ein Schmelzen der Probe beobachtet werden konnte. Zusammengefasst konnte dadurch gezeigt werden, dass Reduktionsreaktionen in den Materialien bei technisch gut beherrschbaren Temperaturen von $800-1400^{\circ} \mathrm{C}$ ablaufen. Die thermische Dissoziation der Materialien scheint hingegen erst bei rund $1000^{\circ} \mathrm{C}$ einzusetzen, wodurch vorgeschaltete thermische Prozessschritte in der LIB-Recycling- kette, beispielsweise zur Deaktivierung der Zellen, einen größeren Spielraum haben ohne Änderungen in den Materialien hervorzurufen.

Während in den grundlagenorientierten Erhitzungsmikroskop-Versuchen das Verhalten der Materialien an sich im Vordergrund stand, wurden in weiteren Versuchsreihen die Besonderheiten des InduRed-Reaktorkonzepts und deren Einfluss auf Transferkoeffizienten speziellerer Elemente in die Produktphasen betrachtet. Dazu wurden unter anderem die Materialien NCA und NMC111 sowie aus in einem dem InduRed-Konzept nachempfundenen Reaktor im Labormaßstab, der sogenannten InduMelt-Anlage (Abb. 5a,b), mit 10 m.- \% Kohlenstoffzugabe eingesetzt und auf knapp $1600^{\circ} \mathrm{C}$ erhitzt.

Die Bedingungen innerhalb des Reaktors (Abb. 5b) entsprechen dabei annähernd jenen der kontinuierlichen Anlage, sie erlaubt jedoch eine weniger aufwendige Durchführung von Vorversuchen. Nachdem das Experiment beendet ist, wird der Reaktor zerlegt und die Produktphasen, Schlacke und Metall, welche am Reaktorboden oder als Ablagerungen an den Grafitwürfeln vorliegen, gesammelt und per ICP-MS analysiert. Dadurch kann am Ende bewertet werden, in welchem Ausmaß sich Kobalt, Nickel, Mangan und 
Abb. 6: Bilder der gesammelten Proben aus dem InduMeltVersuch mit NMC111 bei Zugabevon 10 m.-\% Kohlenstoffpulver. a Schlackenphase, b Metalllegierung [16]

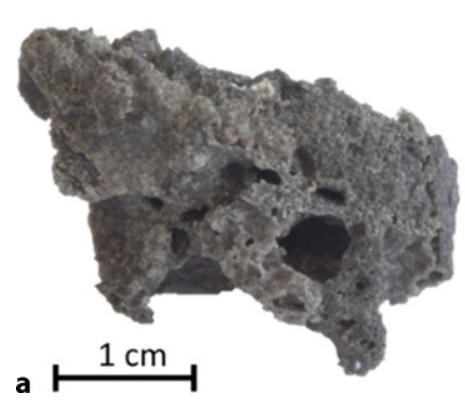

a

Lithium in der Schlacke- und Metallphase (Abb. 6a,b) angereichert haben bzw. ob sie über die Gasphase aus dem Reaktor entfernt wurden. Für eine detaillierte Beschreibung der Versuchsdurchführung und -auswertung wird an dieser Stelle auf die entsprechende Veröffentlichung im MDPI Journal Processes [18] verwiesen.

Zusammengefasst wurden bei den InduMelt-Versuchen mit NMC111 und NCA jeweils ungefähr $550 \mathrm{~g}$ bzw. $520 \mathrm{~g}$ Inputmaterial, bestehend aus dem Kathodenmaterial und $10 \mathrm{~m} .-\%$ Kohlenstoffpulver, in den Reaktor eingesetzt. Nach dem Versuch wurden für NMC11 etwa $240 \mathrm{~g}$ einer Metalllegierung und $38 \mathrm{~g}$ einer Schlacke gefunden, woraus eine $\mathrm{Ge}-$ wichtsreduktion von etwa $46 \%$ resultiert. Bei dem Versuch mit NCA wurden $520 \mathrm{~g}$ eingesetzt und etwa $270 \mathrm{~g}$ Metall bzw. 22 g Schlacke gefunden, wodurch sich eine Gewichtsreduktion von rund $41 \%$ ergibt. Erklären lässt sich die Gewichtsreduktion großteils durch die Bildung von $\mathrm{CO}$ während der Reduktion, wobei der Sauerstoff des Kathodenmaterials und das Reduktionmittel Kohlenstoff verbraucht werden bzw. durch die Entfernung von Lithium und anderen flüchtigen Verbindungen über die Gasphase. Die angestrebte Lithium-Entfernung wurde durch die Analysen der Produktphasen bestätigt. Der Gehalt an Lithium in der Metallphase lag bei unter $0,1 \mathrm{~m} .-\%$, jener in der Schlacke bei etwa $9 \mathrm{~m} . \%$. Aufgrund der geringen Schlackenmenge, welche sich im Versuch gebildet hatte, resultiert daraus eine Lithium-Entfernungsrate von $96 \%$ für NCA bzw. $91 \%$ für NMC.

\section{Zusammenfassung}

Die globalen Anstrengungen zur Verringerung der Treibhausgasemissionen sowie die daraus resultierende steigende Nachfrage nach erschwinglichen Elektrofahrzeugen stellen den Treibstoff für die Entwicklung immer neuer Lithium-lonen-Batterie-Technologien dar. Die damit verbundene, veränderliche chemische Zusammensetzung der Zellen wird in den nächsten Jahrzehnten die Recyclingwirtschaft vor neue Herausforderungen stellen. Sind es in einer ersten Phase in Europa vor allem Batterien vom Typ NCA und NMC, entwickelt sich der Abfallstrom in den nächsten 20-30 Jahren kontinuierlich weiter, wobei zum jetzigen Zeitpunkt noch nicht abzuschätzen ist, welche Wertmetalle neben Lithium in den Batterien der Autos der Zukunft verbaut sein werden. Um trotzdem in der Zukunft erfolgreich, nachhaltig und wirtschaftlich agieren zu können, benöti-

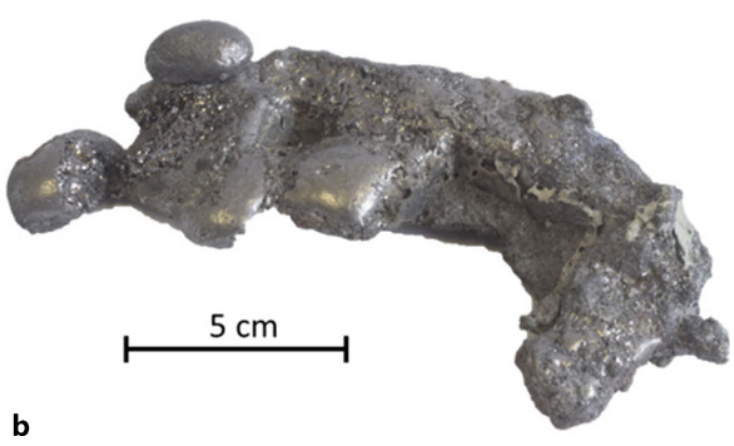

gen Recyclingunternehmen Technologien, welche flexibel auf veränderliche Abfallströme aus in der Elektromobilität eingesetzten LIBs reagieren können. Ein vielversprechender Zugang könnte dabei das vorgestellte InduRed-Konzept sein, welches den Ansatz verfolgt, eine Verschlackung des Lithiums durch entsprechende Reaktionsbedingungen zu unterdrücken und dieses stattdessen separat zurückzugewinnen. In den Vorversuchen in der InduMelt-Anlage wurden dahingehend vielversprechende Ergebnisse erzielt, da es gelang, über $90 \%$ des Lithiums aus dem Reaktor zu entfernen. Diese Separierung ist ein wichtiger Schritt, da dadurch eine wirtschaftliche Rückgewinnung des ansonsten in der Schlacke stark verdünnten Lithiums leichter zu realisieren wäre. Die weiteren Forschungsschwerpunkte liegen in der besseren Charakterisierung der ablaufenden Reaktionen sowie der Anpassung des Reaktorkonzepts an die Bedürfnisse von Abfallströmen aus Alt-LIBs.

Förderung. Dieses Projekt wird mit Mitteln der steiermärkischen Landesregierung und des Klimaschutzfonds der Stadt Graz im Rahmen der 13. Zukunftsfonds Ausschreibung, Green Tech 100 - 1 Earth, 0 Carbon, 0 Waste (PN 1305), gefördert.

\section{ZUKUNF ISFONDS STEIERMARK}

Funding. Open access funding provided by Montanuniversität Leoben.

Open Access Dieser Artikel wird unter der Creative Commons Namensnennung 4.0 International Lizenz veröffentlicht, welche die Nutzung, Vervielfältigung, Bearbeitung, Verbreitung und Wiedergabe in jeglichem Medium und Format erlaubt, sofern Sie den/die ursprünglichen Autor(en) und die Quelle ordnungsgemäß nennen, einen Link zur Creative Commons Lizenz beifügen und angeben, ob Änderungen vorgenommen wurden.

Die in diesem Artikel enthaltenen Bilder und sonstiges Drittmaterial unterliegen ebenfalls der genannten Creative Commons Lizenz, sofern sich aus der Abbildungslegende nichts anderes ergibt. Sofern das betreffende Material nicht unter der genannten Creative Commons Lizenz steht und die betreffende Handlung nicht nach gesetzlichen Vorschriften erlaubt ist, ist für die oben aufgeführten Weiterverwendungen des Materials die Einwilligung des jeweiligen Rechteinhabers einzuholen.

Weitere Details zur Lizenz entnehmen Sie bitte der Lizenzinformation auf http://creativecommons.org/licenses/by/4.0/deed.de.

\section{Literatur}

1. Bundesministerium für Nachhaltigkeit und Tourismus: Integrierter nationaler Energie- und Klimaplan für Österreich, 2019, https://www. 
bmk.gv.at/themen/klima_umwelt/klimaschutz/nat_klimapolitik/ energie_klimaplan.html. Zugegriffen: 04.01.2021

2. Miao, Y.; Hynan, P.; Jouanne, A. von; Yokochi, A.: Current Li-lon Battery Technologies in Electric Vehicles and Opportunities for Advancements, Energies, 12 (2019), Nr. 6, S. 1074, https://doi.org/10.3390/ en 12061074

3. European Commission. Joint Research Centre: Li-ion batteries for mobility and stationary storage applications: scenarios for costs and market growth. Publications Office, 2018. https://doi.org/10. $2760 / 87175$

4. Deutsche Rohstoffagentur: DERA-Rohstoffliste 2019, 2019 https:// www.deutsche-rohstoffagentur.de/DE/Gemeinsames/Produkte/ Downloads/DERA_Rohstoffinformationen/rohstoffinformationen40.pdf?_blob=publicationFile\&v $=5$. Zugegriffen: 04.01.2021

5. Pillot, C.: The Rechargeable Battery Market and Main Trends 2018-2030, Stockage Batterie, Paris, France, 2019

6. Bloomberg NEF: (2019) Electric Vehicle Outlook 2020, 2019, https:// about.bnef.com/electric-vehicle-outlook/. Zugegriffen: 04.01.2021

7. Kraftfahrtbundesamt: (2020) Fahrzeugzulassungen im Dezember 2019 - Jahresbilanz - korrigierte Fassung, 2020, https://www.kba de/DE/Statistik/statistik_inhalt.html. Zugegriffen: 04.01.2021

8. Ziemann, S.; Müller, D. B.; Schebek, L.; Weil, M.: Modeling the potential impact of lithium recycling from EV batteries on lithium demand: A dynamic MFA approach. Resources, Conservation and Recycling, 133 (2018), S. 76-85, https://doi.org/10.1016/j.resconrec. 2018.01.031

9. Fraunhofer-Institut für System- und Innovationsforschung ISI: Gesamt-Roadmap Lithium-ionen-Batterien 2030, 2015, https://www. isi.fraunhofer.de/content/dam/isi/dokumente/cct/lib/GRM-LIB.pdf. Zugegriffen: 04.01.2021

10. Liu, C.; Lin, J.; Cao, H., Zhang, Y., Sun, Z.: Recycling of spent lithiumion batteries in view of lithium recovery: A critical review, Journal of Cleaner Production, 228 (2019), S. 801-813, https://doi.org/10.1016/j. jclepro.2019.04.304

11. Battery recycling: https://csm.umicore.com/en/battery-recycling/emobility. Zugegriffen: 04.01.2021
12. European Commission: Commission staff working document on the evaluation of the Directive 2006/66/EC on batteries and accumulators and waste batteries and accumulators and repealing Directive 91/157/EEC, 2019, https://ec.europa.eu/environment/waste/ batteries/pdf/evaluation_report_batteries_directive.pdf. Zugegriffen: 04.01.2021

13. Schönberg, A.: Mathematische Modellierung metallurgischer Prozesse - Induktive Erwärmung einer Graphitschüttung, PhD Thesis, Lehrstuhl für Thermoprozesstechnik, Leoben: Montanuniversität, 2014

14. Windisch, S.; Ponak, C.; Mally, V.; Raupenstrauch, H.: Phosphorrück gewinnung aus Klärschlammaschen mit dem RecoPhos-Prozess, Österr Wasser- und Abfallw, 72 (2020), Nr. 9-10, S. 421-428, https:// doi.org/10.1007/s00506-020-00696-3

15. Ponak, C.: Carbo-thermal reduction of basic oxygen furnace slags with simultaneous removal of phosphorus via the gas phase, PhD Thesis, Lehrstuhl für Thermoprozesstechnik, Leoben: Montanuniversität, 2019

16. Ponak, C., Mally, V., Windisch, S., Holzer, A., Raupenstrauch, H.: Phosphorus Gasification During The Reduction Of Basic Oxygen Furnace Slags In A Novel Reactor Concept, AML 11 (2020), Nr. 7, S. 20071535, https://doi.org/10.5185/amlett.2020.071535

17. Windisch-Kern, S.; Holzer, A.; Nagovnak, P.; Ponak, C.: Raupenstrauch, H.: Pyrometallurgical recycling of lithium ion batteries: Preliminary experiments to investigate the behaviour of cathode materials under reducing conditions, in Institute of Chemical, Environmental and Bioscience Engineering (ICEBE)TU Wien (Hrsg): Proceedings of the 16. Minisymposium Verfahrenstechnik \& 7 . Partikelforum, Wien, 2020, S. 21-25

18. Windisch-Kern, S.; Holzer, A.; Ponak, C.; Raupenstrauch, H.: Pyrometallurgical Lithium-lon-Battery Recycling: Approach to Limiting Lithium Slagging with the InduRed Reactor Concept. Processes, 9 (2021), Nr. 1, S. 84, https://doi.org/10.3390/pr9010084

Hinweis des Verlags. Der Verlag bleibt in Hinblick auf geografische Zuordnungen und Gebietsbezeichnungen in veröffentlichten Karten und Institutsadressen neutral. 